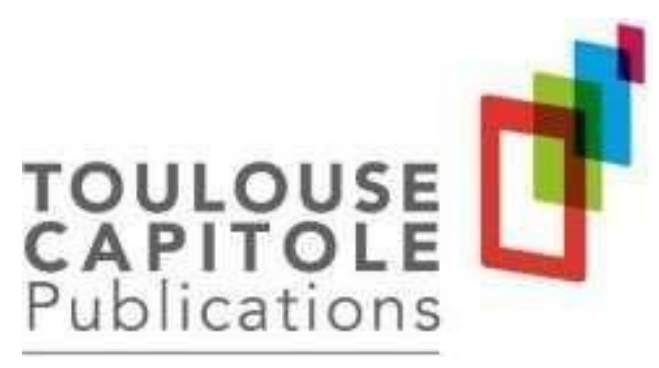

« Toulouse Capitole Publications » est l'archive institutionnelle de l'Université Toulouse 1 Capitole.

L'incidence des services écosystémiques en droit de la responsabilité civile

\author{
JEAN SEVERIN
}

Référence de publication: Jean, Séverin, «L'incidence des services écosystémiques en droit de la responsabilité civile ». Droit et ville, $\mathrm{n}^{\circ}$ 84, 2017. 


\section{L'incidence des services écosystémiques en droit de la responsabilité civile}

L'expression « services écologiques »1 est apparue pour la première fois en droit de la responsabilité à travers la directive du 21 avril 2004, relative à la responsabilité environnementale2, transposée3 aujourd'hui aux articles L. 160-1 et suivants du Code de l'environnement. En effet, l'article L. 161-1-I-4 dispose que « constituent des dommages causés à l'environnement au sens du présent titre les détériorations directes ou indirectes mesurables de l'environnement qui : (...) affectent les services écologiques (...) ». L'insertion de cette expression au sein de la responsabilité environnementale n'est guère étonnante dans la mesure où, comme le souligne Madame Mélodie Fèvre dans sa thèse4, on assiste aujourd'hui à une crise des fonctionnalités des écosystèmes et partant, à une crise des services rendus. Aussi, la responsabilité - environnementale - se présentait comme l'outil juridique efficace, pour ne pas dire «magique », pour assurer la réparation des atteintes à l'environnement et, plus spécialement, de celles portées aux services écosystémiques.

Pourtant, au-delà du faible emploi de la responsabilité environnementale sur le terrain judiciaire 5, c'est surtout son champ d'application restreint qui peut expliquer l'absence de succès de la responsabilité environnementale. Assurément, en limitant cette responsabilité à certaines ressources6, en l'imputant aux seuls exploitants dans le cadre d'une activité professionnelle7, en ne retenant que les préjudices écologiques purs8, en n'envisageant que les faits générateurs du dommage intervenus avant le 30 avril 2007, en excluant les dommages à l'environnement survenus dans certaines circonstances9, il n'est pas étonnant que cette responsabilité environnementale - spéciale - ne soit pas parvenue, du moins pleinement, à satisfaire les objectifs poursuivis au titre desquels figure notamment la remise en état initial des services écologiques10. Toutefois, il ne faudrait pas dépeindre un tableau aussi maussade car la responsabilité environnementale dispose de quelques atouts : une responsabilité quasiment sans faute11, une réparation exclusivement en nature conforme au but poursuivi, l'absence de gravité exigée au titre des atteintes aux services écosystémiques12. Autrement dit, il ne s'agit pas de souhaiter la disparition de ce régime spécial de responsabilité environnementale mais de dire qu'il est insuffisant. Cette carence a sans doute conduit le législateur à réagir en consacrant récemment d'une part, la réparation du préjudice écologique au sein du Code civil13 et d'autre part, en ajoutant, pour consolider l'édifice, une action de groupe en matière environnementale14.

Si l'expression « services écologiques » ou « services écosystémiques » n'est pas expressément employée dans la nouvelle action de groupe, il y a tout lieu de penser que cette action joue pleinement en la matière puisque l'article L. 142-3-1-II du Code de l'environnement traite de tous les préjudices résultant d'un dommage dans les domaines visés par l'article L. 142-2 du même Code. or, étant donné qu'il est question de nature, d'environnement, il est certain que les atteintes aux services écologiques entrent dans le champ d'application de l'action de groupe nouvellement créée. Les choses sont en revanche beaucoup plus claires quand il s'agit de s'intéresser à la responsabilité civile délictuelle tendant à la réparation du préjudice écologique du Code civil. En effet, l'article 1247 du Code civil dispose qu' « est réparable, (...) le préjudice écologique consistant en une atteinte non négligeable (...) aux bénéfices collectifs tirés par l'homme de l'environne- ment ». Bien que l'article $1247 \mathrm{du}$ Code civil n'use pas de l'expression « services écologiques » ou « services écosystémiques », les bénéfices collectifs tirés par l'homme de l'environnement constituent assurément un synonyme dans la mesure où les bénéfices correspondent aux services, aux bienfaits que retire l'homme de l'écosystème entendu plus maladroitement au sein du Code civil par la référence à l'environnement. L'orientation « anthropocentrée »15 du texte ne laisse subsister aucun doute : les 
services écosystémiques entrent dans le champ de la responsabilité civile délictuelle des articles 1246 et suivants du Code civil. Aussi, la question n'est plus de savoir si les services écologiques sont prévus par l'article 1247 du Code précité, mais de se demander si l'insertion du préjudice écologique, constitué notamment par une atteinte aux dits services, influence la responsabilité civile délictuelle.

La réponse pour le signataire est évidente : assurément. Assurément, parce que l'influence des services écosystémiques se retrouve non seulement au stade des conditions de mise en œuvre de la responsabilité civile (I) mais également au stade du régime de responsabilité civile lui-même (II).

\section{L'INCIDENCE DES SERVICES ÉCOSYSTÉMIQUES SUR LES CONDITIONS DE MISE EN OEUVRE DE LA RESPONSABILITÉ CIVILE}

La mise en œuvre de la responsabilité civile exige trois conditions cumulatives : un fait générateur, un dommage et un lien de causalité entre les deux premiers éléments. Or, l'insertion des services écosystémiques au sein de la responsabilité civile est l'occasion de repenser au moins deux de ces conditions fondamentales. D'une part, parce qu'un préjudice exige nécessairement un dommage tandis que ce dernier ne conduit pas assurément à un préjudice, il convient de savoir si les services écosystémiques - les bénéfices collectifs tirés par l'homme de l'environnement - forment un dommage ou un préjudice. Autrement dit, les services écosystémiques sont l'occasion d'affirmer, une fois de plus, la distinction entre le dommage et le préjudice (A). D'autre part, la responsabilité civile a toujours été pensée à partir des faits générateurs : faits personnels, faits des choses, faits d'autrui. Toutefois, il est frappant de constater que la responsabilité civile, en matière de services écosystémiques, se moque du fait générateur ; seule comptant la nature du dommage. Dès lors, cette indifférence interroge à nouveau sur l'opportunité de construire des régimes de responsabilité civile non plus à partir des faits générateurs mais à partir de la nature des dommages. Dès lors, les services écosystémiques sont l'occasion de repenser, peut-être à son détriment, la responsabilité civile elle-même (B).

\section{A. LES SERVICES ÉCOSYSTÉMIQUES AU SERVICE DE LA DISTINCTION ENTRE LE DOMMAGE ET LE PRÉJUDICE}

La rédaction de l'article 1247 du Code civil est l'occasion de mettre en évidence la distinction opérée par une partie de la doctrine entre le dommage et le préjudice16 ; le dommage constituant le seuil de la lésion, le préjudice établissant les conséquences - patrimoniales ou extrapatrimoniales - de la lésion. En effet, l'article 1247 du Code précité dispose qu' « est réparable (...) le préjudice écologique consistant en une atteinte non négligeable aux éléments ou aux fonctions des écosystèmes ou aux bénéfices collectifs tirés par l'homme de l'environnement ॥. Il faudrait alors comprendre que l'atteinte aux éléments des écosystèmes tout comme les atteintes aux fonctions des écosystèmes ou encore aux bénéfices collectifs tirés par l'homme de l'environnement constituent des préjudices écologiques. Pourtant, à bien y regarder, le législateur confond le dommage et le préjudice en mettant sur le même plan l'un et l'autre.

Le préjudice écologique serait d'abord constitué par l'atteinte aux éléments des écosystèmes. Pourtant un écosystème est défini comme « un complexe dynamique formé de communautés de plantes, d'animaux et de micro-organismes et de leur environnement non vivant qui, par leur interaction, forment une unité fonctionnelle »17. Autrement dit, il s'agirait de porter atteinte à un de ces éléments et partant, par exemple, 
à une plante composant un écosystème considéré. Le préjudice écologique serait ensuite une atteinte aux fonctions des écosystèmes, c'est-à-dire aux manifestations fonctionnelles des écosystèmes. Le préjudice écologique pourrait enfin être établi en cas d'atteinte aux bénéfices collectifs tirés par l'homme de l'environnement, c'est-à-dire des services éco- systémiques ou encore des manifestations fonctionnelles des écosystèmes utiles à l'homme. Reprenons alors la distinction entre le dommage et le préjudice soutenue par une partie de la doctrine. Le dommage désigne le seuil de la lésion, c'est-à-dire le bien atteint, tandis que le préjudice vise la détermination des conséquences de la lésion, c'est-à-dire, les utilités perdues du bien du fait de l'atteinte18. Partant, il est possible d'affirmer que l'atteinte aux éléments des écosystèmes ne permet que d'identifier le bien atteint, le dommage, à partir du fait dommageable, l'atteinte aux éléments des écosystèmes. A contrario, l'atteinte aux fonctions ou aux bénéfices collectifs tirés par l'homme des écosystèmes tend à mettre en lumière non plus le dommage mais les préjudices consécutifs à l'atteinte aux éléments des écosystèmes, c'est-à-dire aux fonctions ou utilités perdues, ou du moins dégradées, des écosystèmes. Il ressort de cette analyse que le législateur, à travers l'article 1247 du Code civil, opère une confusion regrettable entre le dommage et le préjudice ; le dommage ne conduisant pas forcément à un préjudice, l'atteinte aux éléments des écosystèmes n'ayant pas pour conséquence inéluctable la dégradation ou la perte d'une fonction des écosystèmes ou d'un service écosystémique. En d'autres termes, s'il est fondamental en responsabilité civile de mettre en exergue le bien atteint, les écosystèmes donc, il est inutile, au titre des conditions de mise en œuvre, de lister les préjudices réparables pour la simple raison que le propre de la responsabilité civile, une fois engagée, a pour objet la réparation de tous les préjudices comme, par exemple, la dégradation ou la perte de fonctions des écosystèmes ou des bénéfices collectifs tirés par l'homme desdits écosystèmes.

En revanche, le législateur, en mettant sur le même plan les atteintes aux fonctions des écosystèmes et les atteintes aux bénéfices collectifs tirés par l'homme, a au moins le mérite de clarifier la distinction entre ces deux préjudices a priori réparables. Madame Fèvre, dans sa thèse, distingue clairement les deux puisque selon elle, « une fonction ne prend la forme d'un service qu'à partir du moment où les pratiques et besoins sociaux reconnaissent ce service comme tel, c'est-à-dire dès lors que la fonction est utile à la satisfaction d'un besoin humain, plus généralement, à l'accomplissement de son bien-être, et non à une entité naturelle »19. Autrement dit, il s'agit de dire que les écosystèmes ont à la fois des utilités intrinsèques - les fonctions des écosystèmes - et des utilités extrinsèques - les bénéfices collectifs tirés par l'homme - parce qu'elles profitent à l'homme et qu'elles sont perçues ainsi par l'homme. Pour le dire encore différemment, un écosystème dispose de fonctions propres qui profitent à d'autres écosystèmes (utilités intrinsèques) ou à l'homme (utilités extrinsèques). Cette distinction fait écho à la proposition faite par le professeur Guillaume Beaussonie de voir dans les services écosystèmiques des utilités extrinsèques d'un bien - les écosystèmes - « dont la particularité est de bénéficier à tous, bien au-delà de leur seul propriétaire, qui ne saurait empêcher son déploiement »20. Aussi, c'est sans doute en raison de cette particularité - utilité bénéficiant à tous - qu'il est difficile pour la responsabilité civile de se saisir de ce préjudice réparable dans la mesure où cette dernière est construite sur l'idée que le préjudice réparable doit être personnel alors que les services écosystémiques constituent des bénéfices collectifs.

Si la dégradation ou la perte de fonctions des écosystèmes, en tant que préjudices réparables, se heurte assurément à l'exigence du caractère personnel en matière de responsabilité civile sauf à considérer, comme le professeur Mireille Bacache, que l'adaptation de la responsabilité civile à l'évolution des besoins indemnitaires doit conduire à se contenter d'exiger un préjudice objectif21, la problématique est la même lorsqu'il s'agit de s'intéresser à la dégradation ou la perte des bénéfices collectifs tirés par l'homme de l'environnement. En effet, comme l'indique le professeur Mustapha Mekki, le préjudice 
écologique peut être concret ou subjectif qu'il soit collectif ou individuel22. Si l'on imagine assez bien qu'une victime puisse, à titre individuel, solliciter, sur le fondement de l'un des faits générateurs de responsabilité civile du Code civil23 (faits personnels, faits des choses ou faits d'autrui), l'indemnisation de son préjudice personnel à la condition de démontrer qu'il a perdu un service écosystémique, c'est-àdire une utilité de son bien qui peut être collective mais qui doit être assurément individuelle, en revanche, les choses se compliquent lorsqu'il est question d'obtenir la réparation de la dégradation ou de la perte des services écosystémiques visés par l'article 1247 du Code civil. En qualifiant les bénéfices de l'environnement comme des bénéfices collectifs, on retombe inexorablement en opposition avec la conception classique de la responsabilité civile exigeant le caractère personnel du préjudice. Pourtant, les articles 1246 et suivants du Code civil n'ont aucunement vocation à réparer des préjudices individuels. Il suffit pour s'en convaincre de lister les personnes habilitées à agir - l'État ou par exemple l'Agence française pour la biodiversité - ou encore le régime de réparation avec notamment l'affectation possible des dommages et intérêts. Aussi, de deux choses l'une : soit la réparation du préjudice écologique au titre duquel figurent les services écosystémiques conduisent effectivement à la déformation ou, à tout le moins, à l'adaptation de la responsabilité civile des articles 1246 et suivants du Code civil ; soit il ne s'agit pas, à proprement parler, de responsabilité civile. En tout état de cause, les services écosystémiques offrent l'opportunité de repenser non plus seulement les conditions de la responsabilité civile mais la responsabilité civile elle-même.

B. LES SERVICES ÉCOSYSTÉMIQUES AU DÉTRIMENT DE LA RESPONSABILITÉ CIVILE ELLE$M \hat{E} M E$

Celles et ceux qui s'intéressent à la responsabilité civile savent qu'elle a toujours su s'adapter aux nécessités indemnitaires de son temps. Il y a finalement deux manières, parfois cumulatives d'ailleurs, de réussir ce tour de force. Soit en jouant sur le régime de responsabilité civile en facilitant, par exemple, l'indemnisation du préjudice corporel ; soit en s'attaquant aux conditions de mise en œuvre de la responsabilité civile. Dans cette dernière hypothèse, il est alors possible, par exemple, de choisir un fait générateur plus facile à rapporter, de reconnaître de nouveaux préjudices réparables ou encore de diminuer l'exigence relative à l'établissement du lien de causalité. Aussi, si l'on veut bien admettre que la responsabilité civile, lorsqu'elle poursuit comme finalité l'indemnisation des préjudices qu'ils soient individuels ou collectifs, demeure de la responsabilité civile alors il importe que ces conditions de mise en œuvre s'adaptent à l'objectif visé, à la condition toutefois que ces conditions demeurent. Dès lors, rien n'interdit le rattachement du préjudice écologique, en l'occurrence la dégradation ou la perte des bénéfices collectifs tirés par l'homme, à la responsabilité civile quand bien même serait-elle une responsabilité civile spéciale comme l'a voulu le législateur en lui dédiant un chapitre spécifique au sein du Code civil. Par conséquent, les services écosystémiques ne sont tant intéressants pour rejeter leur rattachement à la responsabilité civile que pour repenser la responsabilité civile elle-même.

La responsabilité civile a toujours été pensée à partir du fait générateur. Ainsi, le Code civil recense des régimes de responsabilité civile reposant sur des faits personnels, des faits des choses ou encore des faits d'autrui. Pourtant, une partie de la doctrine milite, depuis des décennies, pour la construction de régimes de responsabilité civile fondés non plus sur un fait générateur mais en fonction de la nature du dommage24. Le droit positif le fait en réalité déjà au titre de certains régimes de responsabilité civile puisque, par exemple, la victime d'un accident de la circulation obtiendra assurément l'indemnisation des préjudices consécutifs à un dommage corporel quand bien même elle aurait commis une faute - du moment qu'elle n'est pas inexcusable et cause exclusive de l'accident - alors que la même victime verra 
son indemnisation limitée ou exclue pour les autres dommages25. La détermination d'une ratio légis adaptée à la nature du dommage va dans le sens de l'histoire de la responsabilité civile. D'ailleurs, le projet de réforme de la responsabilité civile de la Chancellerie va dans cette direction dans la mesure où, par exemple, des règles spécifiques sont prévues selon que les préjudices résultent d'un dommage matériel ou corpore126. Cela étant, la prise en compte de la nature du dommage n'est pour l'heure envisagée qu'au stade des régimes de certaines responsabilités civiles et non pas à celui des conditions de mise en œuvre. Pourtant, il apparaît possible, avec l'insertion de la réparation des services écosystémiques, de concevoir une responsabilité civile fondée exclusivement à partir du dommage.

L'idée n'est pas nouvelle puisque Boris Starck, dans sa thèse consacrée à la théorie de la garantie, proposait déjà une hiérarchisation des intérêts protégés - à l'allemande - par le truchement de la nature des dommages27. Depuis, une partie de la doctrine estime que l'atteinte au corps - le dommage corporel donc - est l'intérêt protégé supérieur de telle manière qu'il justifierait un traitement spécifique au profit des victimes. Les autres, en revanche, parce qu'ils constituent des intérêts de moindre importance exigeraient, à la fois ou soit, des conditions de mise de la responsabilité civile davantage drastiques -une faute par exemple - ou un régime de responsabilité civile moins bienveillant comme, par exemple, l'exigence d'une valeur minimale du dommage lorsque ce dernier est matériel28. Ce qui est frappant en matière de services écosystémiques quand il s'agit d'évoquer la responsabilité civile instituée aux articles 1246 et suivants du Code civil, c'est l'absence d'exigence d'un fait générateur spécifique. Autrement dit, la nature du fait générateur n'importe pas puisqu'il suffit qu'un dommage écologique survienne, c'est-àdire l'atteinte à un écosystème, pour mobilier cette responsabilité civile.

En revanche, la rédaction de l'article 1247 du Code civil renseigne sur la place qu'a voulu lui accorder le législateur au titre de la hiérarchisation des intérêts protégés. En effet, cette disposition exige une atteinte « non négligeable » aux éléments des écosystèmes. En d'autres termes, il ne suffit pas qu'un dommage écologique survienne mais un dommage écologique d'une certaine gravité. Si la gravité du dommage n'est pas inconnue en responsabilité civile puisqu'elle permet de différencier le traitement des préjudices au stade de certains régimes de responsabilité - en matière de produits défectueux par exemple - elle n'était, jusqu'à la consécration de la réparation du préjudice écologique dans le Code civil, aucunement requise au titre des conditions de mise en œuvre des régimes de responsabilité civile. Cette nouveauté conduit à affirmer que l'environnement n'est pas - du moins pour la responsabilité civile - l'intérêt protégé le plus important. L'exigence d'une gravité du dommage au stade des conditions de mise en œuvre de la responsabilité civile est condamnable à au moins deux égards. D'une part, comme le suggère Monsieur Huglo, un « petit » dommage peut conduire à de " grands » préjudices tandis qu'un « grand » dommage peut donner lieu à de « petits » préjudices29. D’autre part, la gravité du dommage écologique tend à créer une inégalité de traitement - toujours au stade des conditions de mise en œuvre de la responsabilité civile - puisque la responsabilité civile des articles 1246 et suivants du Code civil ne permet la réparation notamment que de la dégradation ou de la perte des bénéfices collectifs tirés par l'homme de l'environnement lorsque ces préjudices ne sont pas individuels. Or, on peut parfaitement imaginer qu'une victime, pour ces mêmes préjudices, en sollicite l'indemnisation parce qu'ils lui sont personnels et individuels. Si tel est le cas, certes la victime devra se fonder sur un autre régime de responsabilité civile du Code civil, mais la gravité du dommage écologique ne sera pas exigée au stade des conditions de mise en œuvre de la responsabilité civile choisie. Dès lors, cela ne signifie pas que l'intérêt protégé l'écosystème - ait fait l'objet d'une hiérarchisation tandis que cela conduit à une inégalité de traitement des victimes dans la mesure où les bénéfices collectifs titrés par l'homme peuvent être à la fois des utilités extrinsèques collectives et des utilités extrinsèques individuelles. En définitive, les services 
écosystémiques ont assurément une incidence sur les conditions de mise en œuvre de la responsabilité civile. Reste à savoir si tel est également le cas lorsqu'on s'intéresse au régime de la responsabilité civile.

\section{L'INCIDENCE DES SERVICES ÉCOSYSTÉMIQUES SUR LE RÉGIME DE RESPONSABILITÉ CIVILE}

Les services écosystémiques, par le truchement du dommage écologique, ont également une incidence quant au régime de responsabilité civile à au moins deux niveaux. D'une part, lorsqu'il s'agit de s'intéresser aux aspects procéduraux de l'action qui certes ne sont pas rédhibitoires pour l'efficacité de l'action mais qui doivent faire l'objet d'éclaircissement afin d'ailleurs de pouvoir disposer d'un socle cohérent pour prendre en charge le dommage écologique (A). D'autre part, le même constat, avec cette fois-ci beaucoup plus d'inconvénients, doit être dressé quant à la réparation elle-même dont le régime est pour l'heure insatisfaisant ou, tout du moins, peu enclin à donner envie d'agir (B).

\section{A. \\ LES SERVICES ÉCOSYSTÉMIQUES AU STADE DE L'ACTION EN RESPONSABILITÉ CIVILE}

L'article 1248 du Code civil prévoit que « l'action en réparation du préjudice écologique est ouverte à toute personne ayant qualité et intérêt à agir, telle que l'État, l'Agence française pour la biodiversité, les collectivités territoriales et leurs groupements dont le territoire est concerné, ainsi que les établissements publics et les associations agréées ou créées depuis au moins cinq ans à la date d'introduction de l'instance qui ont pour objet la protection de la nature et la défense de l'environnement ». Cette disposition, qui doit notamment permettre l'indemnisation de la dégradation ou de la perte de services écosystémiques, est l'occasion de mettre en évidence les aspects procéduraux de l'action en responsabilité civile30. En effet, à travers l'exigence de la qualité et de l'intérêt agir, c'est la question du cumul des actions qui doit retenir notre attention.

Par principe, le cumul de ces deux conditions procédurales n'est pas exigé dans la mesure où, conformément à l'article 31 du Code de procédure civile, l'intérêt à agir une fois acquis, il n'est pas nécessaire de rechercher une qualité à agir. Ce n'est que par exception, lorsque l'action est dite « attitrée ", que le législateur offre, à des personnes déterminées dépourvues d'intérêt personnel à agir, la qualité à agir pour défendre un intérêt. Or, l'article 1248 du Code précité ne procède aucunement de la sorte puisque qualité et intérêt agir sont requis. L'on ne peut qu'espérer qu'il s'agisse d'une maladresse dans la rédaction de la disposition car on voit mal pourquoi le législateur aurait dressé une liste31 des personnes ayant la qualité à agir pour exiger d'elles qu'elles démontrent en sus un intérêt à agir. Il est donc plus logique de penser que législateur a voulu procéder à une dissociation. D'une part, les personnes visées en disposant de la qualité à agir n'ont pas à démontrer l'intérêt à agir; d'autre part, toutes les autres personnes doivent quant à elles démontrer un intérêt à agir duquel sera déduite la qualité à agir. Pour autant, le recours régulier à une telle action en réparation de préjudices collectifs, sans y adjoindre une action en réparation des préjudices personnels, semble peu probable sauf à considérer que l'altruisme suffira à combattre l'inertie de celles et ceux qui n'ont rien de personnel à y gagner. Aussi, c'est davantage sur un cumul d'actions qu'il faut compter pour tendre notamment à l'indemnisation de la dégradation ou de la perte d'un service écosystémique.

Si l'on met de côté la responsabilité environnementale, qui fait office d'une police administrative peu performante, plusieurs actions civiles peuvent se cumuler puisqu'elles ne poursuivent pas les mêmes fins quand bien même il s'agirait d'obtenir la réparation des préjudices consécutifs à un dommage 
environnemental. En effet, plusieurs intérêts peuvent être lésés pour un même dommage corporel : ceux des personnes qui subissent personnellement un préjudice et qui devront alors agir, soit à titre individuel en recourant à l'une des responsabilités civiles prévues par le Code civil à l'exception de celle consacrée aux articles 1246 à 1252 du Code civil, soit par le truchement de l'action de groupe de l'article L. 142-3$1 \mathrm{du}$ Code de l'environnement; ceux des personnes qui subissent un préjudice collectif et qui devront alors intenter leur action sur le fondement des articles 1246 et suivants du Code civil. C'est à ce stade qu'il est indispensable de procéder à la qualification des préjudices et pour ce faire, pourquoi pas, recourir à une nomenclature des préjudices environnementaux32, afin de bien délimiter les actions possibles. Une fois cette identification réalisée, rien n'interdit le cumul des actions du moins lorsqu'il s'agit de distinguer entre les préjudices collectifs et les préjudices individuels ; la réparation des premiers devant être recherchée sur le fondement des articles 1246 à 1252 du Code civil, les seconds exigeant de recourir soit à une action individuelle fondée sur un fait générateur du Code civil - à l'exception des articles 1246 et suivants précités - soit à une action de groupe portée sur le fondement de l'article 142-3-1 du Code de l'environnement. En revanche, une attention toute particulière doit être portée dans cette dernière hypothèse. Le cumul est possible car parfaitement organisé. En effet, les décisions rendues sur le fondement de l'action de groupe disposent de l'autorité de la chose jugée mais seulement à l'égard des membres du groupe de telle manière qu'elle n'interdit à celles et ceux qui prétendent avoir subi un préjudice personnel d'agir à titre individuel. Plus encore, les membres du groupe ne peuvent se voir opposer l'autorité de la chose jugée de telle sorte qu'ils peuvent encore individuellement agir pour les préjudices personnels qui n'auraient pas été invoqués dans le cadre de la décision fondée sur l'action de groupe. Dès lors, sous réserve d'une délimitation des préjudices réparables et d'une interprétation conforme à la distinction entre l'intérêt et la qualité à agir, le droit s'est doté d'un arsenal juridique opérant pour prendre en charge le dommage environnemental et partant, la dégradation ou la perte des bénéfices collectifs tirés par l'homme de l'environnement. Cela étant, l'étude, au titre du régime de cette responsabilité civile, montre que l'enthousiasme doit être relatif lorsqu'il s'agit de s'intéresser à la réparation des préjudices.

Les atteintes aux bénéfices collectifs tirés par l'homme de l'environnement une fois identifiées et mesurées, le Code civil a fait le choix de hiérarchiser les modes de réparation en privilégiant, au titre de l'article 1249 alinéa 1 du Code civil33, la réparation en nature qui est la forme la plus conforme à l'idée de réparation puisqu'il s'agit de supprimer le préjudice. Aussi, ce n'est qu'à défaut, c'est-à-dire en cas d'impossibilité ou d'insuffisance de la réparation en nature, qu'il est possible de recourir à l'allocation de dommages et intérêts. La réparation en nature n'appelle pas d'observations particulières car elle a le mérite d'être en adéquation avec le préjudice subi. Autrement dit, si la réparation en nature consiste à rétablir le service écosystémique perdu, alors cette responsabilité joue pleinement son rôle.

En revanche, lorsque la réparation se réalise par le truchement de dommages et intérêts, il n'est pas certain que la responsabilité civile permette le rétablissement de la situation de la victime quand bien même, en faisant une entorse au principe de non-affectation des dommages et intérêts34, elle affecterait ces fonds à la réparation de l'environnement. Au-delà de la violation du principe de non- affectation, l'affectation des dommages et intérêts, telle que prévue par le législateur risque de ne pas atteindre l'objectif recherché puisqu'il est à craindre une inadéquation entre le préjudice subi et l'affectation retenue. En effet, l'article 1249 alinéa 2 du Code civil dispose qu' « en cas d'impossibilité ou de droit ou de fait ou d'insuffisance des mesures de réparation, le juge condamne le responsable à verser des dommages et intérêts, affectés à 
la réparation de l'environnement, au demandeur ou, si celui-ci ne peut prendre les mesures utiles à cette fin, à l'État ». Or, comme le souligne à juste titre la professeure Mathilde Hautereau-Boutonnet, l'affectation à une notion si large qu'est « l'environnement » peut conduire à ce que « les dommagesintérêts soient utilisés pour, non pas réparer le préjudice écologique constaté au litige, mais l'environnement plus généralement, au-delà de ce préjudice $» 35$. Le risque est d'autant plus probable en cas de pluralité de demandeurs à l'action. Dans cette hypothèse, il est certain que la somme allouée au titre de la réparation du même préjudice écologique, une fois divisée entre les différents demandeurs, pourra être affectée non pas nécessairement à la réparation du préjudice écologique subi mais à la réparation de l'environnement de sorte qu'il n'est pas impossible, au final, de considérer que c'est davantage le préjudice collectif qu'écologique qui est réparé. Le risque d'une inadéquation entre l'affectation des dommages et intérêts et le préjudice subi est-il réel ? Assurément quand le recours aux dommages et intérêts est conditionné par l'impossibilité de procéder à la réparation en nature ou par l'insuffisance de cette dernière ! Les juges peuvent d'abord se heurter à une impossibilité de fait en raison de la complexité des mesures à mettre en œuvre pour tenter de réparer le préjudice subi ; les juges peuvent ensuite retenir une impossibilité de droit tenant, par exemple, à la séparation des autorités judiciaires et administratives lorsque les mesures envisagées contredisent sévèrement une autorisation administrative qui aurait été délivrée ; les juges disposent enfin d'un pouvoir très étendu dans la mesure où le seul constat de l'insuffisance des mesures prises offre la possibilité d'allouer des dommages et intérêts. Dès lors, il est regrettable que le législateur ne se soit pas donné les moyens de s'assurer que l'affectation des dommages et intérêts soit en conformité avec le préjudice écologique subi car à la complexité de l'appréhension de notions, comme celle de services écosystémiques au stade des conditions de mise en œuvre de la responsabilité civile, il a ajouté la complexité d'un régime difficilement praticable36. Plus encore, le recours aux dommages et intérêts en cas de pluralité de demandeurs pour une même action en responsabilité civile fondée sur les articles 1246 et suivants du Code civil, couplé avec d'autres actions en responsabilité civile recherchant cette fois-ci l'indemnisation de préjudices personnels - par le truchement de l'action de groupe de l'article L. 142-3 du Code de l'environnement ou trouvant un fondement dans un fait personnel, un fait des choses ou d'autrui du Code civil - peut conduire à un imbroglio juridique. Audelà de la difficulté à identifier les chefs de préjudices réparables, c'est l'affectation démultipliée des dommages et intérêts aux différentes victimes qui risque de se heurter au principe de la réparation intégrale si les sommes allouées dépassent le(s) préjudice(s) réparable(s). Or, pour l'heure du moins37, les dommages et intérêts punitifs ne sont pas admis et quand bien même ils le seraient, il conviendrait, au moins pour les actions fondées sur les articles 1246 et suivants du Code civil, qu'ils se justifient non pas eu égard à la gravité du dommage mais eu égard à la gravité du fait générateur nécessairement fautif.

Il ressort de cette analyse que l'intégration des services écosystémiques, par le truchement du préjudice écologique, au sein de la responsabilité civile influence assurément cette dernière soit parce qu'elle oblige à l'adapter, voire la déformer, soit parce qu'elle invite à la repenser. Si pour l'heure cette responsabilité civile semble insuffisamment précisée tant au niveau de ses conditions de mise en œuvre qu'au niveau de son régime pour être efficiente, il faut reconnaître qu'elle ouvre ou ré(ouvre) des réflexions pour matricer une responsabilité civile adaptée aux défis du XXIème siècle au titre desquels figurent prioritairement le maintien du financement de la politique d'indemnisation des préjudices subis et la préservation d'un intérêt bien supérieur encore au préjudice corporel : l'environnement. Pour ce faire, de nombreuses voies sont possibles en dehors mais aussi au-dedans de la responsabilité civile. Il est rassurant de voir que la responsabilité civile s'adapte, créé de nouveaux outils comme ici en consacrant - à l'occasion du préjudice écologique - une véritable action substantielle et autonome en cessation de l'illicite38, prospecte en 
imaginant les outils de demain à l'image de la reconnaissance des dommages et intérêts punitifs.

\section{Notes de bas de page}

1. ou « services écosystémiques » dans la mesure où le présent ouvrage les traite comme des synonymes. En ce sens, voir Glossaire.

2. Directive 2005/35/CE du Parlement Européen et du Conseil du 21 avril 2004 sur la responsabilité environnementale en ce qui concerne la prévention et la réparation des dommages environnementaux.

3. Par la loi n ${ }^{\circ}$ 2008-757 du ler août 2008 relative à la responsabilité environnementale et à diverses dispositions d'adaptation au droit communautaire dans le domaine de l'environnement.

4. Fèvre M., Les services écologiques et le droit. Une approche juridique des systèmes complexes, Thèse, 2016, p. 52.

5. Doit-on peut-être citer un arrêt récent rendu par la Cour de cassation qui refusa, au visa de l'article L. 162-1 du Code l'environnement, de retenir la responsabilité environnementale d'une société organisant des randonnées motorisées au motif que la cour d'appel n'avait pas recherché « si l'activité professionnelle de la société (...) faisait partie de celles permettant de retenir une réparation du dommage causé à l'environnement » (Cass. 3ème civ., 29 sep. 2016, n 15-20048).

6. Cf. art. L. 161-1-I-4 ${ }^{\circ}$ C. env.

7. Cf. art. L. 162-1 C. env.

8. Cf. art. L. 162-2 C. env.

9. Cf. art. L. 161-2 C. env.

10. Cf. art. L. 161-9 C. env.

11. Cf. art. L. $162-1-1^{\circ}$ du Code précité. Cela étant le $2^{\circ}$ du même article exige une faute ou une négligence de l'exploitant.

12. Cf. art. L. 161-1-I- $4^{\circ}$ C. env.

13. Cf. les articles 1246 et $\mathrm{s}$. du Code civil issus de la loi $\mathrm{n}^{\circ} 2016-1087 \mathrm{du} 8$ août 2016 pour la reconquête de la biodiversité, de la nature et des paysages.

14. Cf. spécifiquement l'article L. 142-3-1 du Code de l'environnement issu de la loi n 2016-1547 du 18 novembre 2016 de modernisation de la justice du XXIème siècle.

15. Fèvre M., op. cit., p. 37.

16. «Le dommage, désigne, à proprement parler, la lésion subie, qui s’apprécie au siège de la lésion, tandis que le préjudice, qui est la conséquence de la lésion, apparaît comme l'effet ou la suite du dommage : une atteinte à l'intégrité physique, c'est-à-dire un dommage corporel, peut ainsi engendrer des préjudices patrimoniaux (...) et des préjudices extrapatrimoniaux (...) », Le Tourneau $\mathrm{Ph}$. et al, Droit de la responsabilité civile et des contrats, Régimes d'indemnisation, Dalloz-action, 2017-2018, $\mathrm{n}^{\circ} 1304$. Pour une démonstration plus complète, voir les $n^{\circ} 1304$ et ss. du même ouvrage.

17. Cf. glossaire Ecosystème(s).

18. La théorie moderne de la propriété (théorisée par les professeurs Zénati-Castaing F. et Revet Th., Les biens, PUF, 3e ed., 2008) la définit de deux manières. D’une part, elle est objectivement définie en ce sens qu'il s'agit de la qualité qu'a un bien d'appartenir à une personne. Cette conception invite à revenir sur la notion de bien. Pour la doctrine de la propriété renouvelée, une chose ne devient un bien, sous réserve de la Loi, que parce qu'il est utile de se l'approprier. D'autre part, elle est subjectivement définie comme le rapport exclusif entre un sujet de droit et un bien dont le rapport permet à son titulaire de retirer toutes les utilités que le bien permet. Aussi, si l'on veut bien admettre que la propriété, structure de tous 
les droits subjectifs, est protégée par la responsabilité civile (Cf. Jean S., La protection des droits subjectifs par la responsabilité civile, Thèse, Toulouse, 2012) en sus de l'exercice d'actions spécifiques, alors on peut retrouver une concordance parfaite entre d'une part, le dommage et le bien, objet du droit subjectif, et d'autre part, le préjudice et l'utilité. Ainsi, le dommage permet de désigner en responsabilité le bien atteint tandis que le préjudice autorise à identifier les utilités dégradées ou perdues du bien en raison de l'atteinte.

19. Fèvre M., op. cit., p. 49.

20. Beaussonie G., « La qualification juridique des services écosystémiques ».

21. Bacache M., « L'action de groupe en matière environnementale », EEI n 3, Mars 2017, étude 8, $\mathrm{n}^{\circ} 24$ et ss.

22. Mekki M., « Responsabilité civile et droit de l'environnement. - Vers un droit spécial de la responsabilité civile environnementale ?», Responsabilité civile et assurances, $\mathrm{n}^{\circ}$ 5, Mai 2017, dossier 4, $\mathrm{n}^{\circ} 17$.

23. A l'exception des articles 1246 à 1252 du Code civil.

24. Starck B., Essai d'une théorie générale de la responsabilité civile considérée en sa double fonction de garantie et de peine privée, thèse, éd. Rodstein, 1947.

25. Art. 3 et 5 de la loi $\mathrm{n}^{\circ} 85-677$ du 5 juillet 1985 tendant à l'amélioration de la situation des victimes d'accidents de la circulation et à l'accélération des procédures d'indemnisation.

26. Cf. les articles 1267 et suivants du projet de réforme de la Chancellerie présenté le 13 mars 2017.

27. Starck B., op. cit.

28. C'est déjà le cas en matière de produits défectueux où le dommage doit avoir une valeur supérieure à 500 euros.

29. Huglo Ch., « La difficile application de la réparation du préjudice écologique devant le juge », EEI, $n^{\circ} 6$, Juin 2017, dossier 15, n $^{\circ} 3$.

30. Il n'est pas question ici de le développer dans cette contribution mais il est à noter également un risque de confusion entre la recevabilité et le bien-fondé de l'action. En ce sens, cf. Mekki M., op. cit., $\mathrm{n}^{\circ} 11$.

31. A priori non exhaustive par l'utilisation de la locution « tel que ».

32. Neyret L. et Martin G.-J., Nomenclature des préjudices environnementaux, LGDJ, 2012.

33. Art. 1249, alinéa 1 du Code civil : «La réparation du préjudice écologique s'effectue par priorité en nature $\gg$.

34. Cass. crim., 22 fév. 1995, Bull. crim. 1995, n 77. Le juge excéderait ses pouvoirs s’il décidait de l'affectation des dommages et intérêts alloués à la victime. La solution procède non seulement de la fonction des dommages et intérêts car ces derniers ont pour objectif de fournir à la victime les moyens de se procurer des satisfactions de remplacement, mais aussi du principe de libre gestion par chacun de son patrimoine : Cass. 2ème civ., 8 juil. 2004, Bull. civ. 2004, II, $\mathrm{n}^{\circ} 391$ : « le principe de la réparation intégrale n'implique pas de contrôle sur l'utilisation des fonds alloués à la victime qui conserve leur libre utilisation ». Le projet de réforme de la responsabilité civile de la Chancellerie va également dans ce sens puisque l'article 1264 dispose que « la victime est libre de disposer des sommes allouées ».

35. Hautereau-Boutonnet M., «Quelle action en responsabilité civile pour la réparation du préjudice écologique ? », EEI, $\mathrm{n}^{\circ}$ 6, Juin 2017, dossier 14, $\mathrm{n}^{\circ} 20$.

36. Pour quelques solutions pour faire face à ces difficultés, cf. Hautereau-Boutonnet M., ibid., $\mathrm{n}^{\circ} 23$.

37. Le projet de réforme de la responsabilité civile de la Chancellerie présenté le 13 mars 2017 le prévoit à l'article 1266-1.

38. L'article 1252 du Code civil dispose qu' « indépendamment de la réparation du préjudice 
écologique, le juge, saisi d'une demande en ce sens par une personne mentionnée à l'article 1248, peut prescrire les mesures raisonnables propres à prévenir ou faire cesser le dommage ». 